\title{
Generalized $q$-Bernstein-Schurer Operators and Some Approximation Theorems
}

\author{
M. Mursaleen and Asif Khan \\ Department of Mathematics, Aligarh Muslim University, Aligarh 202002, India \\ Correspondence should be addressed to M. Mursaleen; mursaleenm@gmail.com
}

Received 19 May 2013; Accepted 30 July 2013

Academic Editor: Simone Secchi

Copyright (C) 2013 M. Mursaleen and A. Khan. This is an open access article distributed under the Creative Commons Attribution License, which permits unrestricted use, distribution, and reproduction in any medium, provided the original work is properly cited.

We study statistical approximation properties of $q$-Bernstein-Shurer operators and establish some direct theorems. Furthermore, we compute error estimation and show graphically the convergence for a function $f$ by operators and give its algorithm.

\section{Introduction and Preliminaries}

In 1987, Lupaş [1] introduced the first $q$-analogue of Bernstein operator and investigated its approximating and shapepreserving properties. Another q-generalization of the classical Bernstein polynomials is due to Phillips [2]. After that many generalizations of well-known positive linear operators, based on $q$-integers, were introduced and studied by several authors. Recently the statistical approximation properties have also been investigated for $q$-analogue polynomials. For instance, in [1] $q$-analogues of Bernstein-Kantorovich operators; in [3] q-Baskakov-Kantorovich operators; in [4] $q$-Szász-Mirakjan operators; in $[5,6] q$-Bleimann, Butzer and Hahn operators; in [7] $q$-analogue of Baskakov and Baskakov-Kantorovich operators; in [8] q-analogue of Szász Kantorovich operators; in $[9,10] q$-analogue of Stancu-Beta operators; and in [11] $q$-Lagrange polynomials were defined and their classical approximation or statistical approximation properties were investigated.

Schurer [12] introduced the following operators $L_{m, p}$ : $C[0, p+1] \rightarrow C[0,1]$ defined for any $m \in \mathbb{N}$ and any function $f \in C[0, p+1]:$

$$
\begin{array}{r}
L_{m, p}(f ; x)=\sum_{k=0}^{m+p}\left(\begin{array}{c}
m+p \\
k
\end{array}\right) x^{k}(1-x)^{m+p-k} f\left(\frac{k}{m}\right), \\
x \in[0,1] .
\end{array}
$$

Recently, Muraru [13] introduced the $q$-analogue of these operators and investigated their approximation properties and rate of convergence using modulus of continuity. Note that Radu [14] has also used q-intgers to define and study the approximation properties of the q-analogue of Kantorovich operators.

In this paper, we study the statistical approximation properties by $q$-Bernstein-Schurer operators. We also give some direct theorems.

We recall certain notations of $q$-calculus. Let $q>0$. For any $k \in \mathbb{N}_{0}:=\{0\} \cup \mathbb{N}$, the $q$-integer $[k]_{q}$ is defined by

$$
[k]_{q}:= \begin{cases}\frac{\left(1-q^{k}\right)}{(1-q)}, & q \neq 1, \\ k, & q=1,\end{cases}
$$

and the $q$-factorial $[k]_{q}$ ! by

$$
[k]_{q} !:= \begin{cases}{[k]_{q}[k-1]_{q} \cdots[1]_{q},} & k \geq 1, \\ 1, & k=0 .\end{cases}
$$

Also, the $q$-binomial coefficients are defined by

$$
\left[\begin{array}{l}
n \\
k
\end{array}\right]_{q}:=\frac{[n]_{q} !}{[k]_{q} ![n-k]_{q} !} .
$$

Details on $q$-integers can be found in [14]. 
Muraru [13] introduced the following operators known as the generalized $q$-Bernstein-Schurer operators. For any $m \in$ $\mathbb{N}, p$ a fixed positive integer, and $f \in C[0, p+1]$,

$$
\begin{aligned}
& L_{m, p}^{*}(f ; q ; x) \\
& =\sum_{k=0}^{m+p}\left[\begin{array}{c}
m+p \\
k
\end{array}\right]_{q} x^{k} \prod_{s=0}^{m+p-k-1}\left(1-q^{s} x\right) f\left(\frac{[k]_{q}}{[m]_{q}}\right), \\
& x \in[0,1] \text {. }
\end{aligned}
$$

We note the following properties as in [13] for $L_{m, p}^{*}(f ; q ; x)$.

Lemma 1. For $x \in[0,1], 0<q \leq 1$,

$$
L_{m, p}^{*}(1 ; q ; x)=1 \text {. }
$$

Lemma 2. For $x \in[0,1], 0<q \leq 1$,

$$
L_{m, p}^{*}(t ; q ; x)=x \frac{[m+p]_{q}}{[m]_{q}} .
$$

Lemma 3. For $x \in[0,1], 0<q \leq 1$,

$$
L_{m, p}^{*}\left(t^{2} ; q ; x\right)=\frac{[m+p]_{q}}{[m]_{q}^{2}}\left([m+q]_{q} x^{2}+x(1-x)\right) \text {. }
$$

Lemma 4. Let $q \in(0,1)$. Then for $x \in[0,1]$

$$
L_{m, p}^{*}(t-x ; q ; x)=x\left(\frac{[m+p]_{q}}{[m]_{q}}-1\right) .
$$

Lemma 5. Let $q \in(0,1)$. Then for $x \in[0,1]$

$$
\begin{aligned}
L_{m, p}^{*}\left((t-x)^{2} ; q ; x\right) & \\
= & x^{2}\left(\frac{\left([m+p]_{q}-[m]_{q}\right)^{2}-[m+p]_{q}}{[m]_{q}^{2}}\right) \\
& +x \frac{[m+p]_{q}}{[m]_{q}^{2}} .
\end{aligned}
$$

\section{Statistical Approximation}

In this section we obtain the Korovkin type weighted statistical approximation properties for these operators defined in (5). Korovkin type approximation theory [15] has also many useful connections, other than classical approximation theory, in other branches of mathematics (see Altomare and Campiti [16]).

First we recall the concept of statistical convergence for sequences of real numbers which was introduced by Fast [17] and further studied by many others.
Let $K \subseteq \mathbb{N}$ and $K_{n}=\{j \leq n: j \in K\}$. Then the natural density of $K$ is defined by $\delta(K)=\lim _{n} n^{-1}\left|K_{n}\right|$ if the limit exists, where $\left|K_{n}\right|$ denotes the cardinality of the set $K_{n}$.

A sequence $x=\left(x_{j}\right)$ of real numbers is said to be statistically convergent to $L$ provided that for every $\epsilon>0$ the set $\left\{j \in \mathbb{N}:\left|x_{j}-L\right| \geq \epsilon\right\}$ has natural density zero; that is, for each $\epsilon>0$,

$$
\lim _{n} \frac{1}{n}\left|\left\{j \leq n:\left|x_{j}-L\right| \geq \epsilon\right\}\right|=0 .
$$

In this case, we write $s t-\lim _{n} x_{n}=L$. Note that every convergent sequence is statistically convergent but not conversely, even unbounded sequence may be statistically convergent. For example, let $u=\left(u_{m}\right)$ be defined by

$$
u_{m}= \begin{cases}1, & \text { if } k \text { is a square } \\ 0, & \text { otherwise }\end{cases}
$$

Then $s t-\lim u_{m}=0$, but $u$ is not convergent.

Recently the idea of statistical convergence has been used in proving some approximation theorems, in particular, Korovkin type approximation theorems by various authors, and it was found that the statistical versions are stronger than the classical ones. Authors have used many types of classical operators and test functions to study the Korovkin type approximation theorems which further motivate continuation of this study. After the paper of Gadjiev and Orhan [18], different types of summability methods have been deployed in approximation process, for example, [19-23]. Recently, $A$ statistical convergence has been used to the summability of Walsh-Fourier series [24].

Let $C_{B}[0, p+1]$ be the space of all bounded and continuous functions on $[0, p+1]$. Then $C_{B}[0, p+1]$ is a normed linear space with $\|f\|=\sup _{x \geq 0}|f(x)|$. Let $w$ be a function of the type of modulus of continuity. The principal properties of the function are the following:

(i) $w$ is a nonnegative increasing function on $[0, p+1]$,

(ii) $\lim _{\delta \rightarrow 0} w(\delta)=0$.

Let $C_{B}[0, p+1]$ be the space of all real valued functions $f$ defined on $[0, p+1]$ satisfying the following condition:

$$
|f(x)-f(y)| \leq w(|x-y|),
$$

for any $x, y \in[0, p+1]$.

We consider a sequence $q=\left(q_{n}\right), q_{n} \in(0,1)$, such that

$$
\lim _{n \rightarrow \infty} q_{n}=1 \text {. }
$$

The condition (14) guarantees that $[n]_{q_{n}} \rightarrow \infty$ as $n \rightarrow \infty$.

Now our first result is as follows.

Theorem 6. Let $\left(L_{m, p}^{*}\right)$ be the sequence of the operators (5), and the sequence $q=\left(q_{m}\right)$ satisfies (14). Then for any function $f \in C_{B}[0, p+1]$,

$$
s t-\lim _{m \rightarrow \infty}\left\|L_{m, p}^{*}\left(f ; q_{m} ; x\right)-f\right\|=0 .
$$


Journal of Function Spaces and Applications

3

Proof. Let $e_{v}=x^{\nu}$ where $v=0,1,2$. Since $L_{m, p}^{*}\left(1 ; q_{m}, x\right)=$ $q_{m}$, therefore we can write

$$
s t-\lim _{m \rightarrow \infty}\left\|L_{m}^{*}\left(1 ; q_{m} ; x\right)-1\right\|=s t-\lim _{n \rightarrow \infty}\left\|e_{0}\right\|\left|q_{m}-1\right|
$$

as

$$
\begin{aligned}
\left\|L_{m, p}^{*}\left(1 ; q_{m} ; x\right)-1\right\| & =\left|L_{m}^{*}\left(1 ; q_{m} ; x\right)-1\right| \\
& =|| e_{0} \|\left|q_{m}-1\right| \leq\left|q_{m}-1\right| .
\end{aligned}
$$

By (14), it can be observed that

$$
s t-\lim _{m \rightarrow \infty}\left\|L_{m, p}^{*}\left(1 ; q_{m} ; x\right)-1\right\|=0 .
$$

Similarly

$$
\begin{aligned}
\left\|L_{m, p}^{*}\left(t ; q_{m} ; x\right)-x\right\| & =\left\|e_{2}\right\|\left|\frac{q_{m}[m+p]_{q_{m}}^{2}}{[m]_{q_{m}}^{2}}-1\right| \\
& \leq\left|\frac{[m+p]_{q}}{[m]_{q}}-1\right| .
\end{aligned}
$$

For a given $\epsilon>0$, let us define the following sets:

$$
\begin{gathered}
U=\left\{m:\left\|L_{m, p}^{*}\left(t ; q_{m} ; x\right)-x\right\| \geq \epsilon\right\}, \\
U^{\prime}=\left\{m: \frac{[m+p]_{q_{m}}}{[m]_{q_{m}}}-1 \geq \epsilon\right\} .
\end{gathered}
$$

It is obvious that $U \subseteq U^{\prime}$; it can be written as

$$
\begin{aligned}
\delta(\{k & \left.\left.\leq m:\left\|L_{m, p}^{*}\left(t ; q_{m} ; x\right)-x\right\| \geq \epsilon\right\}\right) \\
& \leq \delta\left(\left\{k \leq m: \frac{[m+p]_{q_{m}}}{[m]_{q_{m}}}-1 \geq \epsilon\right\}\right) .
\end{aligned}
$$

By using (14), we get

$$
s t-\lim _{m \rightarrow \infty}\left(\frac{[m+p]_{q_{m}}}{[m]_{q_{m}}}-1\right)=0 .
$$

Therefore

$$
\delta\left(\left\{k \leq m: \frac{[m+p]_{q_{m}}}{[m]_{q_{m}}}-1 \geq \epsilon\right\}\right)=0,
$$

and we have

$$
s t-\lim _{n \rightarrow \infty}\left\|L_{m, p}^{*}\left(t ; q_{m} ; x\right)-x\right\|=0 .
$$

Lastly, we have

$$
\begin{aligned}
& \left\|L_{m, p}^{*}\left(t^{2} ; q_{m} ; x\right)-x^{2}\right\| \\
& =\left\|e_{2}\right\|\left(\frac{q_{m}[m+p]_{q_{m}}^{2}}{[m]_{q_{n}}^{2}}-\frac{q[m+p]_{q_{m}}}{[m]_{q_{m}}^{2}}-1\right) \\
& \quad+\left\|e_{1}\right\|\left(\frac{q_{m}[m+p]_{q_{m}}}{[m]_{q_{m}}^{2}}\right) \leq\left|\frac{q_{m}[m+p]_{q}^{2}}{[m]_{q}^{2}}-1\right| .
\end{aligned}
$$

Therefore by (25), we get

$$
\begin{aligned}
& \left\|L_{m, p}^{*}\left(t^{2} ; q_{m} ; x\right)-x^{2}\right\| \\
& \leq\left|\frac{1}{q_{m}^{2}}\left(\frac{[m+p+1]_{q_{m}}^{2}}{[m]_{q_{m}}^{2}}\right)-1\right| \\
& \quad+\left|\frac{1}{q_{m}^{2}}\left(\frac{\left(q_{m}+2\right)[m+p+1]_{q}}{[m]_{q}^{2}}\right)\right|+\mid \frac{1}{q_{m}^{2}}\left(\frac{1+q_{m}}{[m]_{q}^{2}}\right) .
\end{aligned}
$$

Now, if we choose

$$
\begin{gathered}
\alpha_{m}=\frac{1}{q_{m}^{2}}\left(\frac{[m+p+1]_{q_{m}}^{2}}{[m]_{q_{m}}^{2}}\right)-1, \\
\beta_{m}=\frac{q_{m}+2}{q_{m}^{2}}\left(\frac{[m+p+1]_{q}}{[m]_{q}^{2}}\right), \\
\gamma_{m}=\frac{1}{q_{m}^{2}}\left(\frac{1+q_{m}}{[m]_{q}^{2}}\right),
\end{gathered}
$$

then, by (14), we can write

$$
s t-\lim _{m \rightarrow \infty} \alpha_{m}=0=s t-\lim _{m \rightarrow \infty} \beta_{m}=s t-\lim _{m \rightarrow \infty} \gamma_{m} .
$$

Now for given $\epsilon>0$, we define the following four sets:

$$
\begin{gathered}
U=\left\{m:\left\|L_{m, p}^{*}\left(t^{2} ; q_{n} ; x\right)-x^{2}\right\| \geq \epsilon\right\}, \\
U_{1}=\left\{m: \alpha_{m} \geq \frac{\epsilon}{3}\right\}, \\
U_{2}=\left\{m: \beta_{m} \geq \frac{\epsilon}{3}\right\}, \\
U_{3}=\left\{m: \gamma_{m} \geq \frac{\epsilon}{3}\right\} .
\end{gathered}
$$

It is obvious that $U \subseteq U_{1} \cup U_{2} \cup U_{3}$. Then we obtain

$$
\begin{aligned}
& \delta\left(\left\{k \leq m:\left\|L_{m, p}^{*}\left(t^{2} ; q_{m} ; x\right)-x^{2}\right\| \geq \epsilon\right\}\right) \\
& \leq \delta\left(\left\{k \leq m: \alpha_{m} \geq \frac{\epsilon}{3}\right\}\right)+\delta\left(\left\{k \leq m: \beta_{m} \geq \frac{\epsilon}{3}\right\}\right) \\
&+\delta\left(\left\{k \leq m: \gamma_{m} \geq \frac{\epsilon}{3}\right\}\right) .
\end{aligned}
$$

Using (28), we get

$$
s t-\lim _{m \rightarrow \infty}\left\|L_{m, p}^{*}\left(t^{2} ; q_{m} ; x\right)-x^{2}\right\|=0 .
$$

Since

$$
\begin{aligned}
& \left\|L_{m, p}^{*}\left(f ; q_{m} ; x\right)-f\right\| \\
& \leq\left\|L_{m, p}^{*}\left(t^{2} ; q_{m} ; x\right)-x^{2}\right\|+\left\|L_{m, p}^{*}\left(t ; q_{m} ; x\right)-x\right\| \\
& \quad+\left\|L_{m, p}^{*}\left(1 ; q_{m} ; x\right)-1\right\|,
\end{aligned}
$$


we get

$$
\begin{aligned}
s t- & \lim _{m \rightarrow \infty}\left\|L_{m, p}^{*}\left(f ; q_{m} ; x\right)-f\right\| \\
\leq & s t-\lim _{m \rightarrow \infty}\left\|L_{m, p}^{*}\left(t^{2} ; q_{m} ; x\right)-x^{2}\right\| \\
& +s t-\lim _{m \rightarrow \infty}\left\|L_{m, p}^{*}\left(t ; q_{m} ; x\right)-x\right\| \\
& +s t-\lim _{m \rightarrow \infty}\left\|L_{m, p}^{*}\left(1 ; q_{m} ; x\right)-1\right\|,
\end{aligned}
$$

which implies that

$$
s t-\lim _{m \rightarrow \infty}\left\|L_{m, p}^{*}\left(f ; q_{m} ; x\right)-f\right\|=0 .
$$

This completes the proof of the theorem.

Remark 7. In the following example, we demonstrate that the statistical version is stronger than the ordinary approximation. Let us write $T_{m, p}^{*}\left(f ; q_{m} ; x\right)=\left(1+u_{m}\right) L_{m, p}^{*}\left(f ; q_{m} ; x\right)$, where the sequence $\left(u_{m}\right)$ is defined by $(12)$. Then under the hypothesis of the previous theorem, we have

$$
s t-\lim _{m \rightarrow \infty}\left\|T_{m, p}^{*}\left(f ; q_{m} ; x\right)-f\right\|=0 .
$$

However, $\lim _{m \rightarrow \infty}\left\|T_{m, p}^{*}\left(f ; q_{m} ; x\right)-f\right\|$ does not exist, since $\left(u_{m}\right)$ is statistically convergent but not convergent.

\section{Direct Theorems}

The Peetre's $K$-functional is defined by

$$
K_{2}(f, \delta)=\inf \left[\left\{\|f-g\|+\delta\left\|g^{\prime \prime}\right\|\right\}: g \in W_{p}^{2}\right],
$$

where

$$
W_{p}^{2}=\left\{g \in C_{B}[0, p+1]: g^{\prime}, g^{\prime \prime} \in C_{B}[0, p+1]\right\} .
$$

By [25], there exists a positive constant $C>0$ such that $K_{2}(f, \delta) \leq C w_{2}\left(f, \delta^{1 / 2}\right), \delta>0$, where the second-order modulus of continuity is given by

$$
\begin{aligned}
w_{2} & \left(f, \delta^{1 / 2}\right) \\
& =\sup _{0<h \leq \delta^{1 / 2}} \sup _{x \in[0, p+1]}|f(x+2 h)-2 f(x+h)+f(x)| .
\end{aligned}
$$

Also for $f \in C_{B}[0, p+1]$ the usual modulus of continuity is given by

$$
w(f, \delta)=\sup _{0<h \leq \delta^{1 / 2}} \sup _{x \in[0, p+1]}|f(x+h)-f(x)| .
$$

Theorem 8. Let $f \in C_{B}[0, p+1]$ and $0<q_{m}<1$ such that $q_{m} \rightarrow 1(m \rightarrow \infty)$. Then for all $n \in \mathbb{N}$ and $p$ fixed, there exists an absolute constant $C>0$ such that

$$
\left|L_{m, p}^{*}\left(f ; q_{m} ; x\right)-f(x)\right| \leq C w_{2}\left(f, \delta_{m}(x)\right),
$$

where

$$
\begin{aligned}
\delta_{m}^{2}(x)= & x^{2}\left(\frac{\left([m+p]_{q}-[m]_{q}\right)^{2}-[m+p]_{q}}{[m]_{q}^{2}}\right) \\
& +x \frac{[m+p]_{q}}{[m]_{q}^{2}} .
\end{aligned}
$$

Proof. Let $g \in W_{2}$. From Taylor's expansion, we get

$$
\begin{aligned}
g(t)= & g(x)+g^{\prime}(x)(t-x) \\
& +\int_{x}^{t}(t-u) g^{\prime \prime}(u) d u, \quad t \in[0, A], A>0,
\end{aligned}
$$

And, by Lemmas 1, 2, and 3, we get

$$
\begin{aligned}
& L_{m, p}^{*}(g ; x)=g(x)+L_{m, p}^{*}\left(\int_{x}^{t}(t-u) g^{\prime \prime}(u) d u, x\right), \\
& \left|L_{m, p}^{*}(g ; x)-g(x)\right| \\
& \quad \leq\left|L_{m, p}^{*}\left(\int_{x}^{t}(t-u) g^{\prime \prime}(u) d u, x\right)\right| \\
& \quad \leq L_{m, p}^{*}\left(\left|\int_{x}^{t}\right|(t-u)|| g^{\prime \prime}(u)|d u|, x\right) \\
& \quad \leq L_{m, p}^{*}\left((t-x)^{2}, x\right)\left\|g^{\prime \prime}\right\| .
\end{aligned}
$$

Using Lemma 5, we obtain

$$
\begin{aligned}
& \left|L_{m, p}^{*}(g ; x)-g(x)\right| \\
& \leq x^{2}\left(\frac{\left([m+p]_{q}-[m]_{q}\right)^{2}-[m+p]_{q}}{[m]_{q}^{2}}\right)\left\|g^{\prime \prime}\right\| \\
& \quad+x \frac{[m+p]_{q}}{[m]_{q}^{2}}\left\|g^{\prime \prime}\right\| .
\end{aligned}
$$

On the other hand, by the definition of $L_{m, p}^{*}(f ; q ; x)$, we have

$$
\left|L_{m, p}^{*}(f ; x)\right| \leq\|f\| .
$$

Now

$$
\begin{aligned}
& \left|L_{m, p}^{*}(f ; x)-f(x)\right| \\
& \leq\left|L_{m, p}^{*}(f-g ; x)-(f-g)(x)\right| \\
& \quad+\left|L_{m, p}^{*}(g ; x)-g(x)\right| \\
& \leq\|f-g\|+x^{2}\left(\frac{\left([m+p]_{q}-[m]_{q}\right)^{2}-[m+p]_{q}}{[m]_{q}^{2}}\right)\left\|g^{\prime \prime}\right\| \\
& \quad+x \frac{[m+p]_{q}}{[m]_{q}^{2}}\left\|g^{\prime \prime}\right\| .
\end{aligned}
$$


TABLE 1: Error estimation table.

\begin{tabular}{|c|c|c|c|}
\hline$m($ for $p=30, q=0.9)$ & $\begin{array}{l}\text { Error bound at } \\
\quad x=0.2\end{array}$ & $\begin{array}{c}\text { Error bound at } \\
x=0.5\end{array}$ & $\begin{array}{l}\text { Error bound at } \\
\quad x=0.8\end{array}$ \\
\hline 20 & 0.2309 & 1.5709 & 2.7170 \\
\hline 30 & 0.1893 & 1.1865 & 1.5829 \\
\hline 50 & 0.1750 & 1.0835 & 1.3792 \\
\hline
\end{tabular}

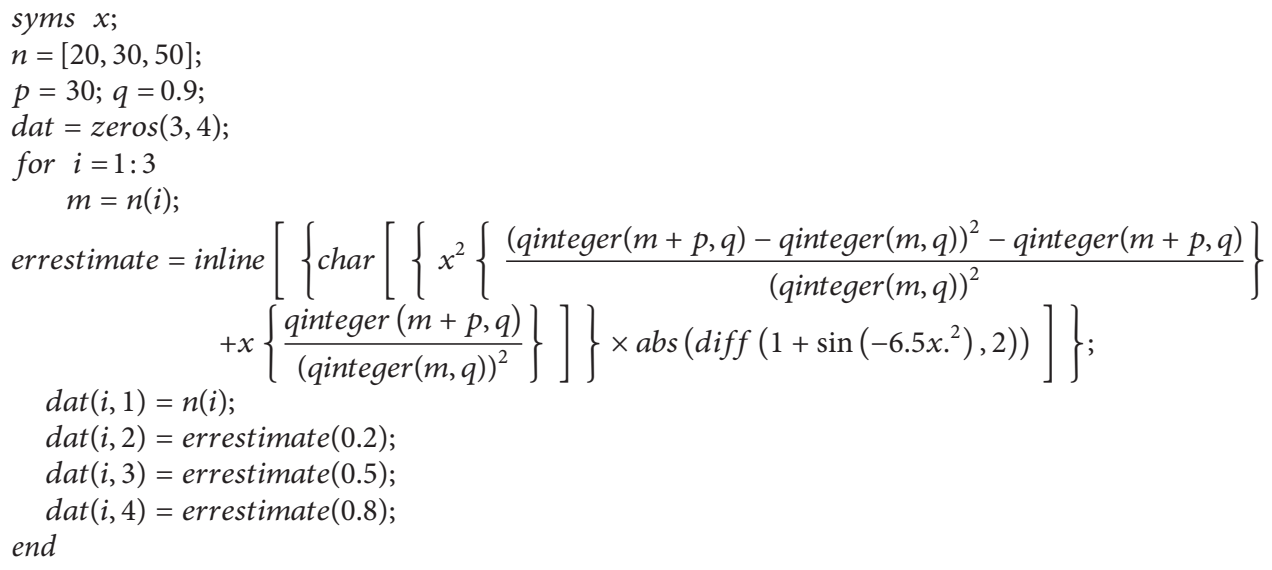

Algorithm 1: Error estimate algorithm.

Hence taking infimum on the right hand side over all $g \in W^{2}$, we get

$$
\left|L_{m, p}^{*}(f ; x)-f(x)\right| \leq C K_{2}\left(f, \delta_{m}^{2}(x)\right) .
$$

In view of the property of $K$-functional, we get

$$
\left|L_{m, p}^{*}(f ; x)-f(x)\right| \leq C w_{2}\left(f, \delta_{m}(x)\right) \text {. }
$$

This completes the proof of the theorem.

Let $C_{B}^{*}[0, p+1]$ be the space of all bounded functions for which $\lim _{x \rightarrow \infty} f(x)$ is finite.

Theorem 9. Let $f \in C_{B}^{*}[0, p+1]$ be such that $f^{\prime}, f^{\prime \prime} \in$ $C_{B}^{*}[0, p+1]$, and the sequence $\left(q_{m}\right)$ satisfies (14). Then the following equality holds:

$$
\lim _{m \rightarrow \infty}[m]_{q_{m}}\left(L_{m, p}^{*}(f ; q ; x)-f(x)\right)=\frac{x(1-x)}{2} f^{\prime \prime}
$$

uniformly on $[0, p+1]$.

Proof. By the Taylor's formula we may write

$$
\begin{aligned}
f(t)= & f(x)+f^{\prime}(x)(t-x)+\frac{1}{2} f^{\prime \prime}(x)(t-x)^{2} \\
& +r(t, x)(t-x)^{2},
\end{aligned}
$$

where $r(t, x)$ is the remainder term and $\lim _{t \rightarrow x} r(t, x)=0$. Applying $L_{m, p}^{*}(f ; q ; x)$ to $(50)$, we obtain

$$
\begin{aligned}
{[m]_{q_{m}} } & \left(L_{m, p}^{*}(f ; q ; x)-f(x)\right) \\
= & {[m]_{q_{m}}\left(L_{m, p}^{*}(t-x ; q ; x) f^{\prime}(x)\right) } \\
& +[m]_{q_{m}}\left(L_{m, p}^{*}\left((t-x)^{2} ; q ; x\right)\right) \frac{f^{\prime \prime}(x)}{2} \\
& +[m]_{q_{m}} L_{m, p}^{*}\left(r(t, x)(t-x)^{2} ; q ; x\right) .
\end{aligned}
$$

By the Cauchy-Schwartz inequality, we have

$$
\begin{aligned}
& L_{m, p}^{*}\left(r(t, x)(t-x)^{2} ; q ; x\right) \\
& \leq \sqrt{L_{m, p}^{*}\left(r^{2}(t, x) ; q ; x\right)} \\
& \quad \times \sqrt{L_{m, p}^{*}\left(r(t, x)(t-x)^{4} ; q ; x\right)} .
\end{aligned}
$$

Observing that $r^{2}(x, x)=0$ and $r^{2}(\cdot, x) \in C_{B}^{*}[0, p+1]$, then it follows from Theorem 8 that

$$
L_{m, p}^{*}\left(r^{2}(t, x) ; q ; x\right)=r^{2}(x, x)=0,
$$

uniformly with respect to $x \in[0, p+1]$. Now from (52), (53), and Lemma 5, we get

$$
\lim _{m \rightarrow \infty}[m]_{q_{m}} L_{m, p}^{*}\left(r(t, x)(t-x)^{2} ; q ; x\right)=0 .
$$




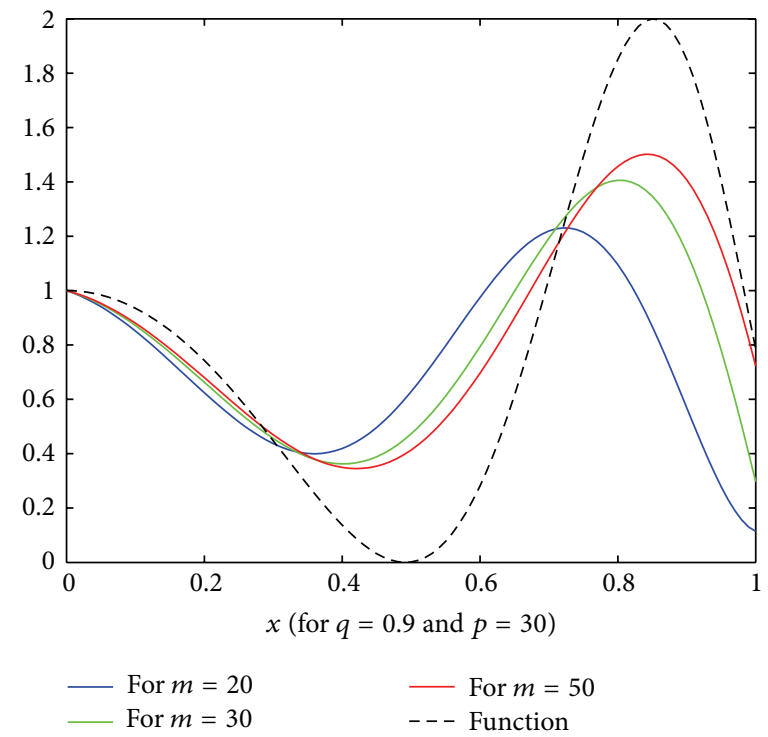

Figure 1

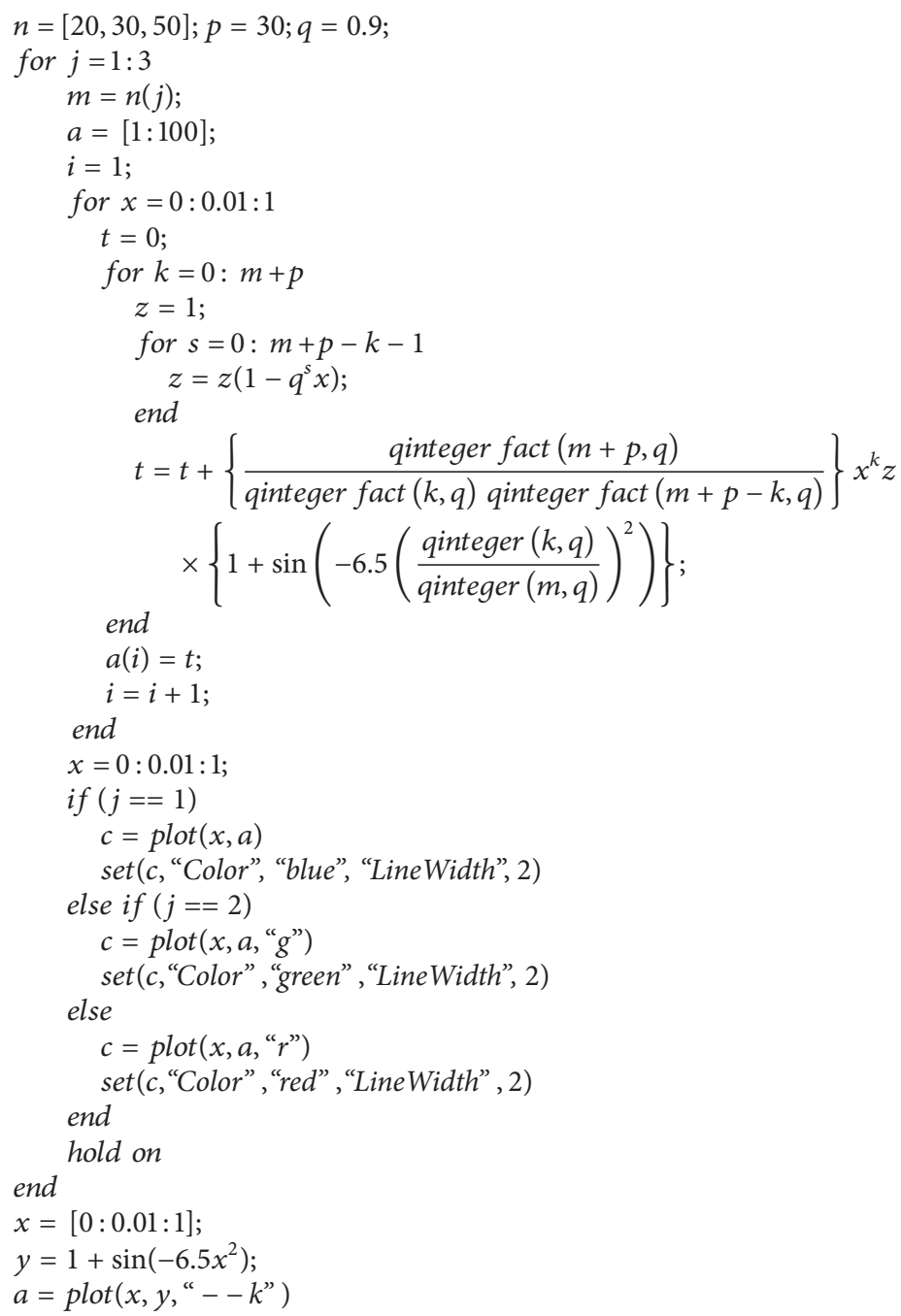

Algorithm 2: For the generalized $q$-Bernstein-Schurer operators. 
Finally using Lemmas 4 and 5, we get the following:

$$
\begin{aligned}
\lim _{m \rightarrow \infty} & {[m]_{q_{m}}\left(L_{m, p}^{*}(f ; q ; x)-f(x)\right) } \\
= & \lim _{m \rightarrow \infty}[m]_{q_{m}}\left(L_{m, p}^{*}(t-x ; q ; x) f^{\prime}(x)\right) \\
& +\lim _{m \rightarrow \infty}[m]_{q_{m}}\left(L_{m, p}^{*}\left((t-x)^{2} ; q ; x\right)\right) \frac{f^{\prime \prime}(x)}{2} \\
& +\lim _{m \rightarrow \infty}[m]_{q_{m}} L_{m, p}^{*}\left(r(t, x)(t-x)^{2} ; q ; x\right) \\
= & \frac{x(1-x)}{2} f^{\prime \prime}(x) .
\end{aligned}
$$

This completes the proof of the theorem.

\section{Example}

Let us take $f(x)=1+\sin \left(-6.5 x^{2}\right)$. We compute error estimation [26] by using modulus of continuity for operators (5) to the function $f(x)=1+\sin \left(-6.5 x^{2}\right)$ shown in Table 1 with the help of MATLAB and Algorithm 1 .

For $m=20,30$, and 50, the convergence of operators (5) to function

$$
f(x)=1+\sin \left(-6.5 x^{2}\right)
$$

is illustrated in Figure 1 with help of Algorithm 2.

\section{References}

[1] A. Lupaş, "A q-analogue of the Bernstein operator," Seminar on Numerical and Statistical Calculus, University of Cluj-Napoca, vol. 9, pp. 85-92, 1987.

[2] G. M. Phillips, "Bernstein polynomials based on the q-integers, The heritage of P.L. Chebyshev," Annals of Numerical Mathematics, vol. 4, pp. 511-518, 1997.

[3] V. Gupta and C. Radu, "Statistical approximation properties of q-Baskakov-Kantorovich operators," Central European Journal of Mathematics, vol. 7, no. 4, pp. 809-818, 2009.

[4] M. Örkcü and O. Doğru, "Weighted statistical approximation by kantorovich type q-Szász-Mirakjan operators," Applied Mathematics and Computation, vol. 217, no. 20, pp. 7913-7919, 2011.

[5] A. Aral and O. Dğru, "Bleimann, Butzer, and Hahn operators based on the q-integers," Journal of Inequalities and Applications, vol. 2007, Article ID 79410, 12 pages, 2007.

[6] S. Ersan and O. Doğru, "Statistical approximation properties of q-Bleimann, Butzer and Hahn operators," Mathematical and Computer Modelling, vol. 49, no. 7-8, pp. 1595-1606, 2009.

[7] N. I. Mahmudov, "Statistical approximation of Baskakov and Baskakov-Kantorovich operators based on the q-integers," Central European Journal of Mathematics, vol. 8, no. 4, pp. 816-826, 2010.

[8] N. Mahmudov and V. Gupta, "On certain $q$-analogue of Szász Kantorovich operators," Journal of Applied Mathematics and Computing, vol. 37, no. 1-2, pp. 407-419, 2011.

[9] A. Aral and V. Gupta, "On the $q$-analogue of Stancu-Beta operators," Applied Mathematics Letters, vol. 25, no. 1, pp. 6771, 2012.
[10] M. Mursaleen and A. Khan, "Statistical approximation properties of modified $q$-Stancu-Beta operators," Bulletin of the Malaysian Mathematical Sciences Society, vol. 36, no. 3, pp. 683690, 2013.

[11] M. Mursaleen, A. Khan, H. M. Srivastava, and K. S. Nisar, "Operators constructed by means of q-Lagrange polynomials and A-statistical approximation," Applied Mathematics and Computation, vol. 219, pp. 6911-6918, 2013.

[12] F. Schurer, "Linear positive operators in approximation theory," Tech. Rep., Mathematical Institute Delft University of Technology, 1962.

[13] C.-V. Muraru, "Note on $q$-Bernstein-Schurer operators," Studia Universitatis Babeş-Bolyai, Mathematica, vol. 56, no. 2, pp. 489495, 2011.

[14] C. Radu, "Statistical approximation properties of Kantorovich operators based on q-integers," Creative Mathematics and Informatics, vol. 17, no. 2, pp. 75-84, 2008.

[15] P. P. Korovkin, Linear Operators and Approximation Theory, Hindustan Publishing Corporation, Delhi, India, 1960.

[16] F. Altomare and M. Campiti, Korovkin Type Approximation Theory and Its Applications, vol. 17 of Studia Mathematica, Walter de Gruyter, Berlin, Germany, 1994.

[17] H. Fast, "Sur la convergence statistique," Colloquium Mathematicum, vol. 2, pp. 241-244, 1951.

[18] A. D. Gadjiev and C. Orhan, "Some approximation theorems via statistical convergence," Rocky Mountain Journal of Mathematics, vol. 32, no. 1, pp. 129-138, 2002.

[19] G. A. Anastassiou, M. Mursaleen, and S. A. Mohiuddine, "Some approximation theorems for functions of two variables through almost convergence of double sequences," Journal of Computational Analysis and Applications, vol. 13, no. 1, pp. 3746, 2011.

[20] M. Mursaleen and A. Alotaibi, "Statistical summability and approximation by de la Vallée-Poussin mean," Applied Mathematics Letters, vol. 24, pp. 320-324, 2012, Erratum: Applied Mathematics Letters, vol. 25, pp. 665, 2012.

[21] S. A. Mohiuddine, A. Alotaibi, and M. Mursaleen, "Statistical summability $(\mathrm{C}, 1)$ and a Korovkin type approximation theorem," Journal of Inequalities and Applications, vol. 2012, article 172, 2012.

[22] M. Mursaleen, V. Karakaya, M. Ertürk, and F. Gürsoy, "Weighted statistical convergence and its application to Korovkin type approximation theorem," Applied Mathematics and Computation, vol. 218, no. 18, pp. 9132-9137, 2012.

[23] M. Mursaleen and A. Kilicman, "Korovkin second theorem via $B$-statistical A-summability," Abstract and Applied Analysis, vol. 2013, Article ID 598963, 6 pages, 2013.

[24] A. Alotaibi and M. Mursaleen, "A-statistical summability of Fourier series and Walsh-Fourier series," Applied Mathematics \& Information Sciences, vol. 6, no. 3, pp. 535-538, 2012.

[25] R. A. Devore and G. G. Lorentz, Constructive Approximation, Springer, Berlin, Germany, 1993.

[26] S. Sezgin and Y. Ertan, "Rate of convergence for Szász type operators including Sheffer polynomials," Studia Universitatis Babeş-Bolyai, Mathematica, vol. 58, pp. 55-63, 2013. 


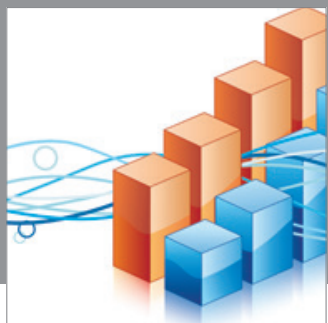

Advances in

Operations Research

mansans

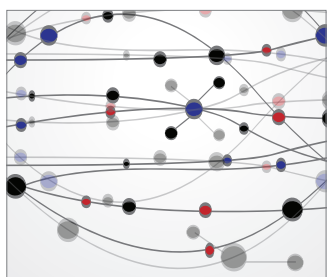

The Scientific World Journal
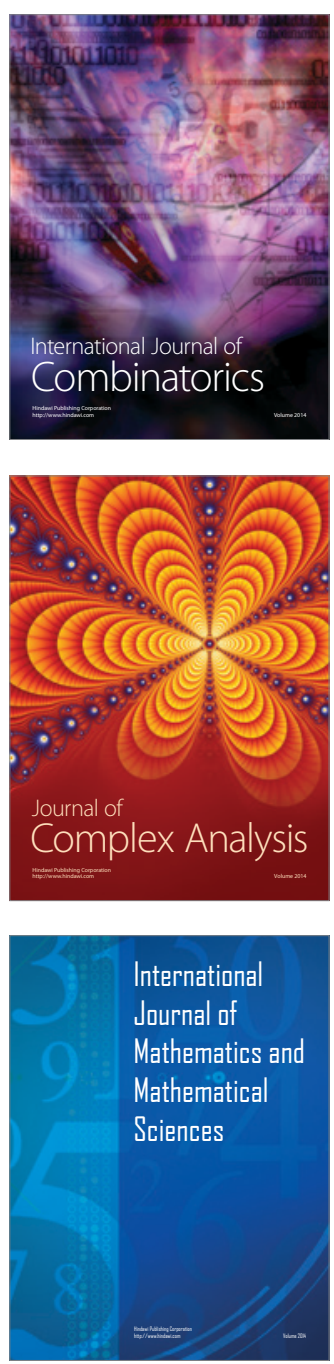
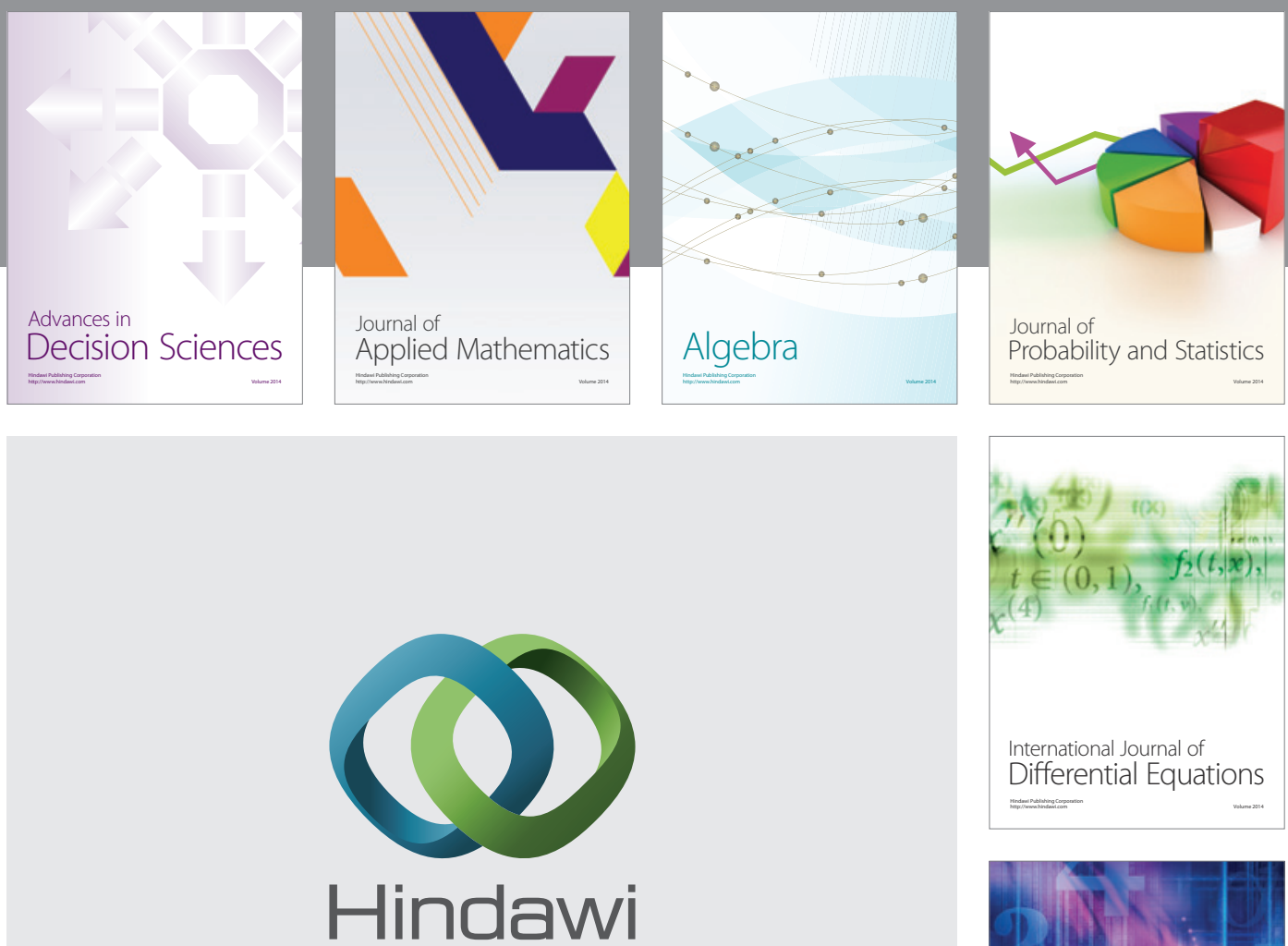

Submit your manuscripts at http://www.hindawi.com
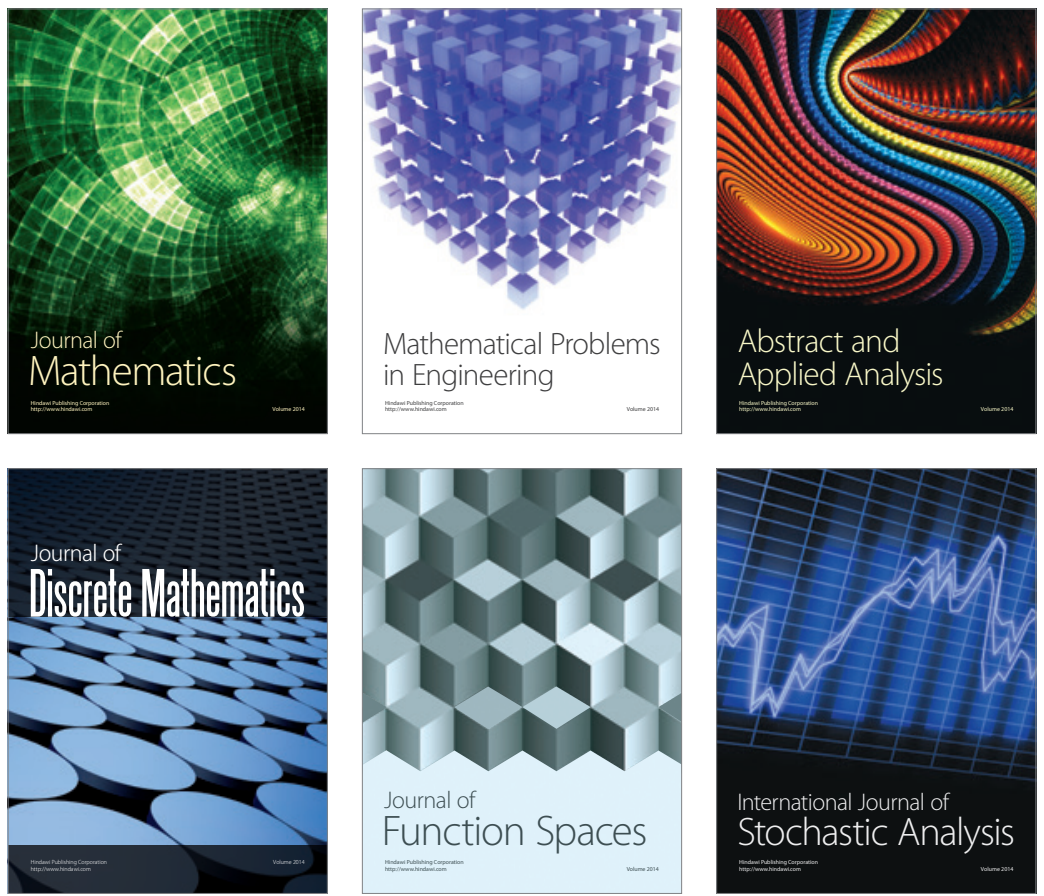

Journal of

Function Spaces

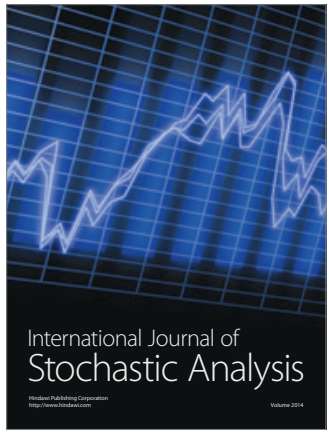

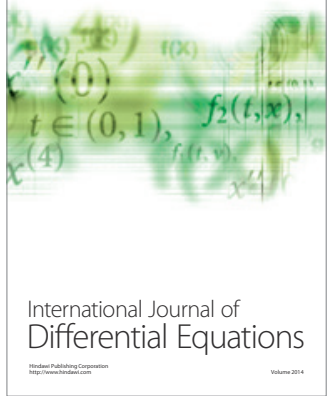
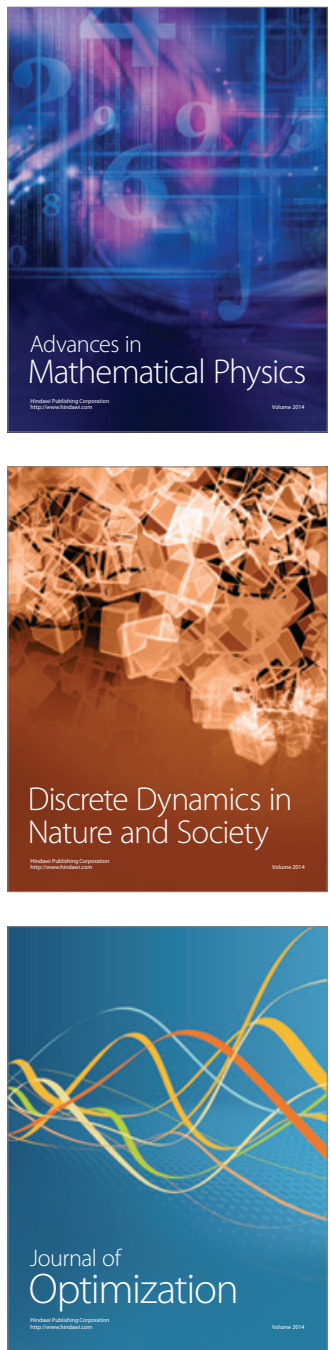